\title{
Recognition of faces in the presence of two-dimensional sinusoidal masks
}

\author{
TODD TIEGER and LEO GANZ \\ Stanford University, Stanford, California 94305
}

\begin{abstract}
The spatial frequencies most relied upon by subjects in a recall task for face recognition were found to lie in the midfrequency range. A linear systems analysis model cannot account for these masking data in terms of retinocortical processing limitations alone. In order to account for the greater disruption of the face recognition task by masks in the range of 2.2 cycles/deg, the existence of unequal filtering of spatial frequency components must be recognized. This unequal filtering may occur either during memory deposition or retrieval of the input stimulus in the recall task or at any time in between.
\end{abstract}

It has been proposed that the human visual system is composed of many independent linear mechanisms, sometimes called "channels," each selectively sensitive to a limited range of spatial frequencies and orientations, and performing, in effect, a Fourier transform on the stimulus input (Campbell \& Robson, 1968). Such a Fourier transform describes the relationship between a luminance distribution and its spatial frequency spectrum (Bracewell, 1965). Utilizing analogies between optical holography and neural signal processing, it has been suggested that pattern recognition involves the linear transduction, storage, and retrieval of a complete or at least partial representation of this two-dimensional transform (LonguetHiggins, 1968; Pollen, Lee, \& Taylor, 1971; Pollen \& Ronner, 1975; Pribram, 1969; Pribram, Nuwer, \& Baron, 1974; Westlake, 1970). The results presented here demonstrate that the process of pattern recognition for human faces is a complex one which may involve intermediary steps, either during memory deposition and/or memory retrieval, in which certain Fourier components are deemphasized relative to others. We may characterize this process as operating in the manner of a higher level selective filter stage in the frequency domain.

The proposal that there are moderately narrow spatial frequency channels in the visual system has received support from a variety of psychophysical and physiological experiments, including the observations of critical band masking (Harmon \& Julesz, 1973; Stromeyer \& Julesz, 1972) and spatial frequency adaptation (Blakemore \& Campbell, 1969; Pantle \& Sekuler, 1968). The independence of these channels has been established by experiments showing that the detection of a test grating is unaffected by the pres-

We thank Eric Sutter and Ben Dawson for their help in designing the grid mask display raster circuitry, George McKee for computation of the Fourier spectra of the face pictures, and Tony Norcia for the stimulus photographs. Support for this work came from NIH Grant 5-ROI EYO 1241-03. ence of a second grating, if the spatial frequencies of the two are sufficiently different (Abadi \& Kulikowski, 1973; Graham \& Nachmias, 1971; Nachmias, Sansbury, Vassilev, \& Weber, 1973; Sansbury, 1977; Stecher, Sigel, \& Lange, 1973; Tolhurst, 1972).

Fourier analytic approaches have only recently begun to extend into the realm of two-dimensional stimuli. Face recognition has been studied with coarsely digitized photographs which, when presented simultaneously with different spatial frequencies of two-dimensional noise, become easier to perceive, depending on how close the noise spectrum is to the digitized face spectrum (Harmon \& Julesz, 1973).

Yet face recognition, presumably requiring the transduction and comparison of a complex list of features or spatial frequency components in all orientations, has been a relatively neglected area of study in vision research (Bradshaw \& Wallace, 1971; Harmon, 1971; Rizzolatti, Umilta, \& Berlucchi, 1971). Nonetheless, the extraordinary ability of humans in this task is undisputed, including not only high recall ability, but a distinctly broad range of stimulus generalizability over time and actual facial transformation (Pittenger \& Shaw, 1975).

The experiment we describe in this paper involves a form of two-dimensional masking at spatial frequencies which lie within the spectrum of normal photographs of faces. We use this technique to measure the relative importance of various spatial frequencies in the process of face recognition.

\section{METHOD}

Our experiment was designed to give a measure of the recognizability of a well-learned set of face pictures when presented simultaneously with grid-like masks of different spatial frequencies. The stimulus population consisted of 300 photographs of faces chosen to represent a homogeneous group. All faces were black and white and chosen from a college yearbook and reproduced under uniform lighting conditions on standard $35-\mathrm{mm}$ 
slides. All pictures were of Caucasians with no glasses, facial hair, or disfigurement. Only the head was shown. Orientation was either full face or three-quarter turn with no more than $10^{\circ}$ tilt of the head. Average luminance of a sample of presented pictures was $20.4 \mathrm{~cd} / \mathrm{m}^{2}$ with contrast $\mathrm{C}=.49$ between the maximum and minimum luminance regions of the photographs, measured by a Gamma 2000 photometer. The experimental apparatus allowed the superimposition of the face stimuli on sine-wave grid masks presented by a Tektronix Model 602 cathode ray display unit with P-11 (purplish-blue) phosphor. The face stimuli were rear-projected on a screen and positioned so as to make a $71-\mathrm{mm}$ square to match the mask display of the same size. The distance to the viewer of $40.6 \mathrm{~cm}$ gave a visual stimulus subtending $10^{\circ}$ visual angle at the retina. A .79-mm-thick piece of clear Plexiglas served as a beam-splitter to superimpose the images of the face stimulus and mask. A Wratten $.8 \mathrm{log}$ unit neutral density filter and bluish filter (unspecified) served to roughly equate luminance and chromaticity of the face pictures with the P-11 display masks. The subject was enclosed in a light-shielding hood containing a chinrest and viewing port for the right eye only. A shutter mechanism controlled the duration of stimulus presentation.

The oscilloscope mask display was provided by a high-frequency raster input with a sinusoidal $\mathrm{z}$-axis modulation. The raster inputs ( $x$ and $y$ axes) were switched at a frame rate of $61 \mathrm{~Hz}$ so as to give a sinusoidal luminance profile grating alternating between horizontal and vertical orientation. The appearance of the display was that of a sinusoidal grid.

In order to assure successful and easy monitoring of the different masks ultimately chosen, photometric measurements and pilot studies were undertaken which resulted in the following choices of experimental parameters: mean luminance of the grid masks was $3.94 \mathrm{~cd} / \mathrm{m}^{2}$, representing the middle of the oscilloscope's measured linearity range; grid contrast was .45 so as not to exceed the linearity range. Grid mask luminance and contrast did not change in any condition.

Six male and four female subjects were each run in two successive sessions for the experiment. During the acquisition phase, which constituted the beginning of each session, subjects were asked to acquaint themselves with a set of 75 face pictures arrayed in a slide tray. During acquisition, the pictures were viewed with the grid mask display turned off. The entire set was presented first at a rate of $2 \mathrm{sec}$ per picture. Then, after a brief rest, the subject paced him- or herself through a second exposure to the same set of pictures. The test phase comprised three randomly ordered conditions, each consisting of 50 subject-initiated .5 -sec exposures of face pictures superimposed on a grid mask of a certain spatial frequency. Spatial frequency conditions used were .54 , $.82,1.1,2.2$, and 3.9 cycles/deg visual angle. The control condition was an unmodulated blank screen with the same mean luminance of $3.94 \mathrm{~cd} / \mathrm{m}^{2}$ as in the other conditions. This is taken to be the "DC" condition.

Figures $1 \mathrm{a}, 1 \mathrm{~b}$, and $1 \mathrm{c}$ show examples of the superimposition of a face photograph and various grid masks. The grids appear roughly as a checkerboard pattern. The spatial frequencies of the grids as they were viewed by the subjects were $1.1 \mathrm{cycles} / \mathrm{deg}$ in Figure 1a, 2.2 cycles/deg in Figure 1b, and 3.9 cycles/deg in Figure 1c. The subjects were instructed to label the pictures as "old" or "new," depending on whether the face presented was recognized as coming from the acquisition phase set of pictures or not. In each of the three test conditions, 25 pictures were duplicates of faces shown in the acquisition phase, and 25 were drawn from the original picture population which the subject had never seen before. The order of faces within each condition was random. The experiment was replicated in a second session the following day with the other set of 150 faces (75 "old" and 75 "new") during acquisition and recall, respectively.

\section{RESULTS AND DISCUSSION}

We calculated the recognizability $\left(\mathrm{d}^{\prime}\right)$ of faces in the presence of grid masks differing only in spatial
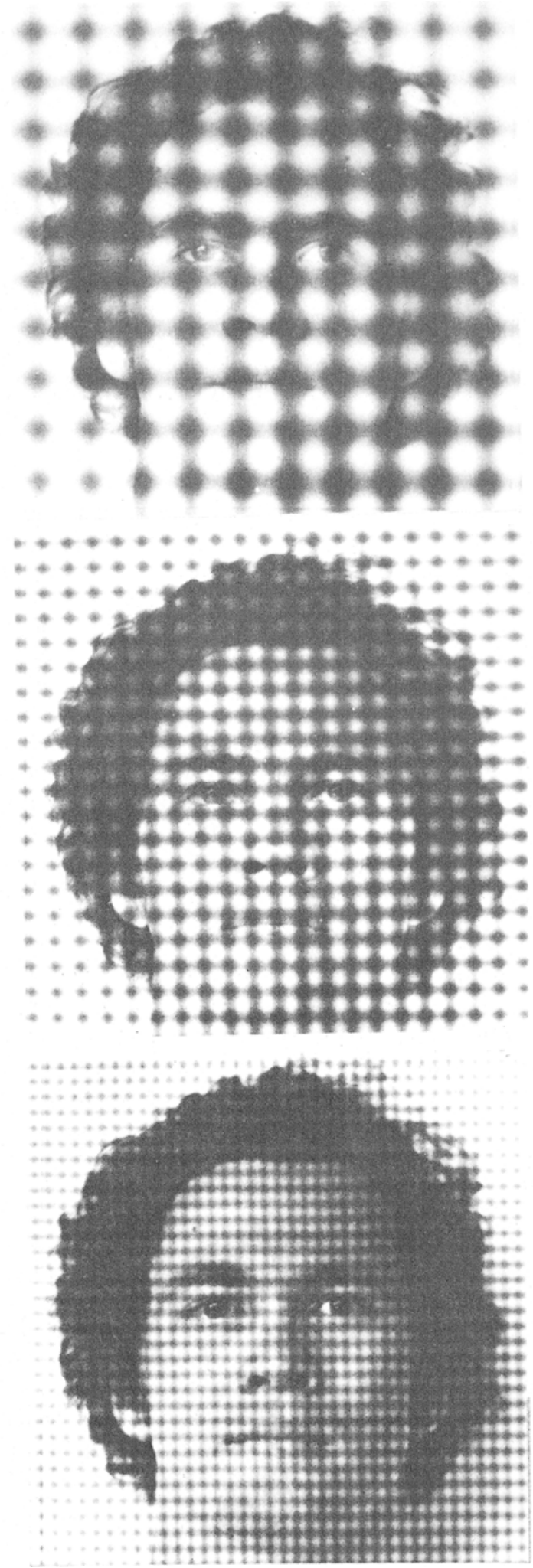

Figure 1. (a) Photograph of simulated stimulus presentation with grid mask of 1.1 cycles/deg. (b) Photograph of simulated stimulus presentation with grid mask of 2.2 cycles/deg. (c) Photograph of simulated stimulus presentation with grid mask of $3.9 \mathrm{cycles} / \mathrm{deg}$. 
frequency. Note that the design is identical to that of a yes-no signal detection experiment with signal probability of .5 (Coombs, Dawes, \& Tversky, 1970). Furthermore, we can use the theory of signal detection to determine if subjects' placement of a criterion varies over the conditions. We wished to determine whether grid masks whose spatial frequencies were near the peak of the human modulation transfer function (e.g., Cornsweet, 1970) would be most effective in masking face recognition. Another theoretical possibility would be that the dimensions of certain spatial features, such as eyes, nose, or mouth (which in our display would correspond to spatial frequencies in the range of 2 cycles $/ \mathrm{deg}$ ), dictated which mask spatial frequency was most effective. Mask effectiveness at some spatial frequency would manifest itself as a lowered recognizability of the faces $\left(d^{\prime}\right)$ at those mask spatial frequencies.

We submitted the results of the $d^{\prime}$ analysis to a repeated measures ANOVA and found them to be highly significant $[\mathrm{F}(5,45)=4.11 ; \mathrm{p}<.001]$. The value of $\beta$ (likelihood ratio at criterion) for all subjects was 1.44 and did not vary significantly from the expected value of $1.0[\mathrm{~F}(5,45)<1 ;$ n.s.].

From these results, we conclude that 2.2 cycles/deg grid masks have the greatest effect on face recognition. Thus, spatial frequency constituents of the face spectrum around this value appear to be relatively more important than other frequencies in the face recognition task. Furthermore, the lack of significant variation in $\beta$ indicates that decision strategy for this task is not affected by changing the spatial frequency of the masking grid. In a general sense, then, these results represent the spatial frequency transfer function for our face-recognition task. We have displayed the results of this analysis in Figure $2 \mathrm{a}$ by inverting $d^{\prime}$ to obtain a measure of relative mask effectiveness for the various grid mask spatial frequencies we used.

In the following section, we present a method of further analyzing our data to ascertain something of the locus of the selective filtering of an input face stimulus. ${ }^{1}$

Perhaps the differences in masking efficacy described in the results above are attributable to the fact that all spatial frequencies are not equally represented in a face. To examine this possibility, we chose five faces from the stimulus population at random and subjected them to a digital Fourier analysis. ${ }^{2}$

Since masking affects primarily the spectral components of similar orientation (Blakemore \& Nachmias, 1971; Campbell, Cleland, Cooper, \& Enroth-Cugell, 1968; Mansfield, 1974; Mitchell, Freeman, \& Westheimer, 1967), we were most interested in comparing our data with the Fourier spectrum of the faces along the vertical and horizontal meridians-the orientation of our grid masks. The average meridional spectrum for our sample is shown in Figure $2 b$. The meridional
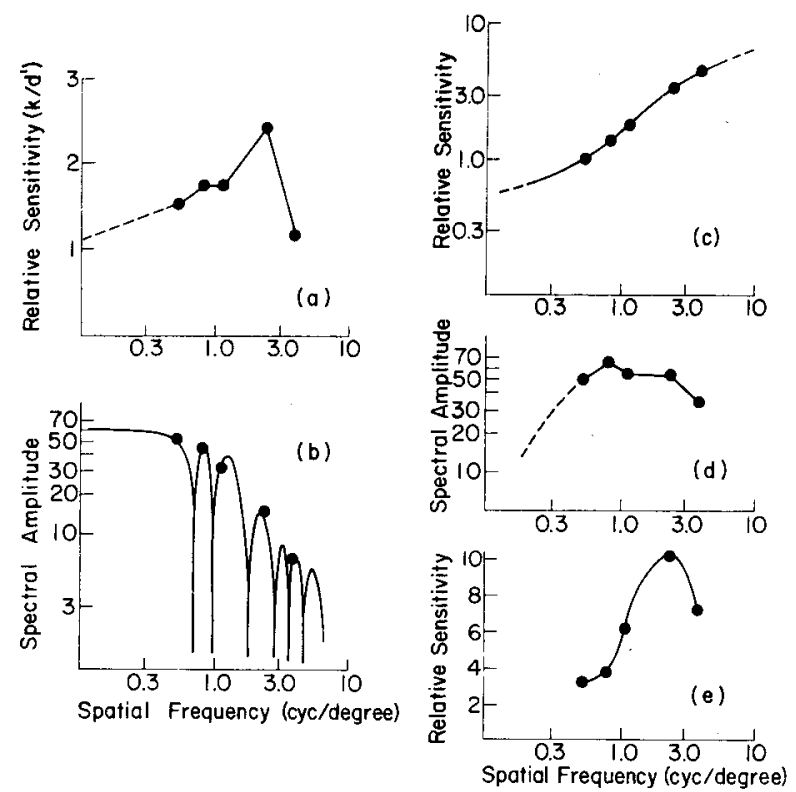

Figure 2. (a) Relative sensitivity of faces $\left(k / d^{\prime}\right)$ to the spatial frequencies of grid masking used. The ordinate is scaled so that the control condition equals 1.0. The scaling constant, $k=1.508$. Data are from 10 subjects. (b) Sample meridional average spectrum of a Fourier transformed face picture. The curve is freely drawn between the sampled data points. (c) Portion of the human modulation transfer function, from Cornsweet (1970, p. 341). (d) Peripheral sensory output stage for the meridional spectrum of Figure $2 \mathrm{~b}$. The control (DC) condition approaches zero and is henceforth omitted in the model considerations. (e) Derived attentional filter showing relative sensitivity biasing beyond peripheral processing. The curve is freely drawn.

spectrum for each picture was an average of the positive $\mathrm{X}$-and $\mathrm{Y}$-axis Fourier coefficients taken from the full two-dimensional transform spectrum.

Figure $2 c$ shows a portion of the human modulation transfer function (MTF) which represents the relative sensitivity of the human visual system in transducing different spatial frequency gratings. Generally speaking, this MTF approximates the transfer characteristics of retinocortical processing (Cornsweet, 1970). If we multiply the meridional Fourier spectrum of a face by the MTF, the product depicts the output of the retinocortical filtering stage which may then be transmitted for later stages of processing; a typical product of this sort is displayed in Figure 2d. A comparison of Figure $2 \mathrm{~d}$ with Figure 2a shows that this peripheral transfer of face spectral input does not parallel the recognition data. More specifically, we would expect the two curves to be quite similar in shape if all spatial frequencies were being equally weighted in the task of face recognition (and the previously mentioned assumption of the comparability of measures holds). It is clear, however, that there are discrepancies between Figures $2 \mathrm{a}$ and $2 \mathrm{~d}$. For example, a large reduction of masking effectiveness is apparent in Figure $2 a$ at 
3 cycles/deg, which is not evident to as great an extent in the spectral output from retinocortical processing of a face photograph in our conditions (Figure 2d) at the same spatial frequency. Therefore, we believe that either during memory deposition of the face representation in the acquisition phase or during the retrieval of that information in the test phase, an unequal weighting of the various spatial frequency components of the input face has occurred. If we continue to pursue the linear systems approach one step further, we may conceive of the intermediary weighting of components as a second-level spatial frequency filter. We may calculate this filter stage's weighting values by dividing the function in Figure $2 \mathrm{a}$ with the meridional face spectrum amplitudes in Figure $2 \mathrm{~d}$ point by point. Figure $2 \mathrm{e}$ contains the results of the computation.

The statement that no such higher level filter exists reduces to the null hypothesis that curve $2 \mathrm{e}$ values are constant over the spatial frequencies measured. An analysis of variance of the data from the five pictures whose spectra we transformed in the manner described shows that the fluctuations shown are, in fact, statistically significant $[F(4,16)=17.64 ; p<.001]$. This result shows that the weighting of spatial frequency components is not accounted for by retinocortical processing limitations. A higher level filter is required to adequately describe the masking data.

Our results indicate that subjects weight certain middle-range spatial frequencies more heavily than either high or low frequencies in recalling faces. Other researchers have reported a similar heavier weighting of midfrequency components in other pattern-recognition tasks. Ginsburg (1973) has shown that certain Gestalt factors of closure, proximity, similarity, etc., as well as certain spatial illusions can be predicted by assuming that the visual system emphasizes medium and low spectral components. Along a similar vein, Bodis-Wollner (1972) has found that, prior to recovery, neurological patients with presumed damage in striate cortex show little, if any, loss in acuity (very high spatial frequency information). However, they do show a substantial loss on contrast sensitivity in the intermediate to high spatial frequency range-a loss which results in impairment in such pattern-recognition tasks as reading. It thus seems that the activation of intermediate spatial frequency channels is more important to pattern perception than the activation of very high spatial frequency channels.

It is not clear whether unequal deposition, storage, retrieval, or task-dependent biasing in the selection of spatial frequency components (Carpenter \& Ganz, 1972 ) is reflected in the masking data we have presented. Retinocortical spatial frequency transduction alone cannot account for our findings. However, our later analysis of the data indicates that the linear systems approach can describe these data with the hypothesis of a higher level spatial frequency filter selectively tuned to midspatial frequency components at some stage of the face recognition-recall process. We point out that the use of a hierarchical model of spatial frequency processing is not unique to our formulation in this paper. Sequential, multiple-channel spatial frequency models of visual perception have also been employed recently in accounting for data on suprathreshold visual texture processing (Harvey \& Gervais, 1978).

Originally, linear systems approaches to the study of visual information processing stressed the potential which these methods offered for a parsimonious explanation of the data of numerous studies (cf. Campbell \& Robson, 1968; Cornsweet, 1970). Subsequently, the proponents of holographic models of visual processing (Pribram, Nuwer, \& Baron, 1974) have made similar claims to the effect that enhanced parsimony would obtain with their model.

The data we have presented argue that at least one higher level linear filter stage is necessary to account for such complex perceptual processes as in face recognition. We suspect that further empirical investigation of the locus of such a filter's action in the recall process may necessitate positing yet other levels of spatial frequency filtering. If this were to be the case, the potential complexity of such a model could develop to equal and perhaps surpass that of visual perception models which are phrased in terms of the spatial stimulus domain as opposed to the spatial frequency domain.

\section{REFERENCES}

AвADI, R. V., \& Kulıkowski, J. J. Linear summation of spatial harmonics in human vision. Vision Research, 1973, 13, 16251628 .

Blakemore, C., \& Campbell, F. W. On the existence of neurones in the human visual system selectively sensitive to the orientation and size of retinal images. Journal of Physiology, $1969,203,237-260$.

Blakemore, C., \& Nachmias, J. The orientation specificity of two visual aftereffects. Journal of Physiology, 1971, 213, $157-174$.

Bodis-Wolnner, I. Visual acuity and contrast sensitivity in patients with cerebral lesions. Science, 1972, 178, 769-771.

Bracewell, R. N. The Fourier transform and its applications. New York: McGraw-Hill, 1965.

Bradshaw, J., \& Wallace, G. Models for the processing and identification of faces. Perception \& Psychophysics, 1971, 9, 443-447.

Campbell, F. W., Cleland, B., Cooper, G., \& EnrothCugels, C. The angular selectivity of visual cortical cells to moving targets. Journal of Physiology, 1968, 198, 237-250.

Campbell, F. W., \& Robson, J. G. Application of Fourier analysis to the visibility of gratings. Journal of Physiology, $1968,197,551-556$.

Carpenter, P., \& Ganz, L. An attentional mechanism in the analysis of spatial frequency. Perception \& Psychophysics, $1972,12,57-60$. 
Coombs, C., Dawes, R., \& Tversky, A. Mathematical psychology. Englewood Cliffs, N.J: Prentice-Hall, 1970.

Cornsweet, T. N. Visual perception. New York: Academic Press, 1970.

Ginsburg, A. P. Pattern recognition techniques suggested from psychological correlates of a model of the human visual system. IEEE Transactions on Aerospace and Electronics, 1973, 9, 625.

Graham, N., \& Nachmias, J. Detection of grating patterns containing two spatial frequencies: A comparison of single channel and multiple channel models. Vision Research, 1971, 11, 251-259.

Harmon, L. D. Some aspects of recognition of human faces. Springer-Verlag: New York, 1971. (Pamphlet not in circulation.)

Harmon, L. D., \& Julesz, B. Masking in visual recognition: Effects of two-dimensional visual noise. Science, 1973, 180, 1194-1197.

Harvey, L. O., \& Gervais, M. J. Visual texture perception and Fourier analysis. Perception \& Psychophysics, 1978, 24, 534-542.

Longuet-Higgins, H. C. Holographic model of temporal recall. Nature, 1968, 217, 104.

MANSFIELD, R. J. W. Neural basis of orientation perception in primate vision. Science, 1974, 186, 1133-1135.

Mitchell, D., Freeman, R. \& Westheimer, G. Effect of orientation on the modulation sensitivity for interference fringes on the retina. Journal of the Optical Society of America, 1967, 57, 246-249.

Nachmias, J., Sansbury, R. V., Vassilev, A., \& Weber, A. Adaptation to square-wave gratings: In search of the elusive third harmonic. Vision Research, 1973, 13, 1335-1342.

Pantle, A., \& Sekuler, R. Size-detecting mechanisms in human vision. Science, 1968, 162, 1146-1148.

Pittenge R, J. B., \& Shaw, R. E. Aging faces as viscal-elastic events: Implications for a theory of non-rigid shape perception. Journal of Experimental Psychology: Human Perception and Performance, 1975, 104, 374-382.

Pollen, D, A., Lee, J., \& Taylor, J. How does the striate cortex begin the reconstruction of the visual world? Science, $1971,173,74-77$.

Pollen, D. A., \& Ronner, S. F. Periodic excitability changes across the receptive fields of complex cells in the striate and parastriate cortex of the cat. Journal of Physiology, 1975, 245, 667-697.

Pribram, K. H. The neurophysiology of remembering. Scientific American, 1969, 220, 73-89.

Pribram, K. H., Nuwer, M., \& Baron, R. The holographic hypothesis of memory structure in brain function and perception. In R. C. Atkinson, D. H. Krantz, R. C. Luce, \& P. Suppes (Eds.), Contemporary developments in mathematical psychology (Vol. II). San Francisco: Freeman, 1974.
Rizzolatti, G., Umilta, C., \& Berlucchi, G. Opposite superiorities of the right and left cerebral hemispheres in discriminative reaction time to physiognomic and alphabetical material. Brain, 1971, 94, 431-442.

Sansbury, R. V. Contrast increment detection of the squarewave third harmonic: Some support for the hypothesis of independent spatial frequency interactions. Vision Research, 1977, 17, 307-309.

Singleton, R. C. Algorithm \#338-ALGOL procedures for the fast Fourier transform. Communications of the $A C M, 1968$, 11, 773-776.

Stecher, S., Sigel, C., \& Lange, R. V. Composite adaptation and spatial frequency interactions. Vision Research, 1973, 13, 2527.2531

Stromeyer, C., \& Julesz, B. Spatial-frequency masking in vision: Critical bands and spread of masking. Journal of the Optical Society of America, 1972, 62, 1221-1232.

TolhurST, D. J. Adaptation to square-wave gratings: Inhibition between spatial frequency channels in the human visual system. Journal of Physiology, 1972, 226, 231-248.

WESTLAKE, P. R. The possibilities of neural holographic processes within the brain. Kybernetik, 1970, 7, 129-153.

\section{NOTES}

1. We shall assume, for this purpose, that our measure of relative recognition sensitivity, $1 / \mathrm{d}^{\prime}$ is proportional to other, more traditional measures of detection threshold contrast sensitivity. While we recognize the potential for being misguided in this assumption, it does seem a likely guess that an increase or decrease in contrast sensitivity at threshold of detection may be alternatively represented by a measure of the inverse of detectability for a given contrast of a stimulus. In any case, the validation of the comparability of such differing measures may be sought empirically in later work.

2. The optical processing equipment at the Stanford Artificial Intelligence Lab enabled us to convert a sample picture of a face photograph into a series of analog voltages and then into a digital representation as a $256 \times 256$ byte core image with 6 bits' intensity level per byte. Due to space limitations in available core, we had to average $2 \times 2$ byte squares in order to load the packaged fast Fourier transform program (Singleton, 1968) with the resultant $128 \times 128$ byte picture and produce the desired transform. The Nyquist high-frequency cutoff was thus 64 lines per picture width which at our viewing distance equals 6.4 cycles/deg visual angle.

(Received for publication November 16, 1978; revision accepted June 5, 1979.) 